\title{
Pan-cancer analysis reveals the role of long non-coding RNA LINC01614 as a highly cancer-dependent oncogene and biomarker
}

\author{
DINGDING WANG ${ }^{1}$, HONG ZHANG $^{1}$, XIAOLIAN FANG ${ }^{2}$, DINGFANG CAO ${ }^{1}$ and HONGGANG LIU ${ }^{1}$ \\ ${ }^{1}$ Department of Pathology, Beijing Tongren Hospital, Capital Medical University, Beijing 100730; \\ ${ }^{2}$ Department of Otolaryngology, Head and Neck Surgery, Beijing Children's Hospital, \\ Capital Medical University, National Center for Children's Health, Beijing 100045, P.R. China
}

Received October 4, 2019; Accepted April 16, 2020

DOI: $10.3892 / \mathrm{ol} .2020 .11648$

\begin{abstract}
Long intergenic non-coding RNA 1614 (LINC01614) is highly expressed in several malignant tumor types, suggesting that it may act as an oncogene. However, the specific roles of LINC01614 in malignant tumors have remained elusive. To examine the expression pattern of LINC01614 in various malignancies, a comprehensive pan-cancer analysis was performed using public databases, including 53 normal tissue types and 32 cancer datasets with samples from 9,091 patients. The results were validated using reverse transcription-quantitative PCR analysis of tissue specimens from patients. LINC01614 expression was upregulated in most malignant tumors, thus demonstrating diagnostic potential. Furthermore, upregulation of LINC01614 was associated with poor overall survival in the majority of cases. However, the association with clinical outcome was highly cancer-dependent; LINC01614 appeared to be an oncogene and diagnostic/prognostic biomarker in cancers of the digestive, respiratory, nervous and endocrine systems, as well as breast and head and neck cancer, but not in the cancers of the reproductive system or some of the urinary system. High LINC01614 expression was also markedly associated with the epithelial-mesenchymal transition (EMT) and associated signaling pathways. Overall, the present results suggest that LINC01614 is an EMT-associated oncogene that influences the metastasis and prognosis of several cancers, thus highlighting its potential as a novel diagnostic and prognostic marker.
\end{abstract}

\section{Introduction}

Despite considerable progress in cancer diagnosis and treatment, 18.1 million new cancer cases were detected and 9.6 million cancer-associated deaths occurred worldwide in

Correspondence to: Professor Honggang Liu, Department of Pathology, Beijing Tongren Hospital, Capital Medical University, 1 Dongjiaominxiang Street, Beijing 100730, P.R. China

E-mail: liuhg1125@163.com

Key words: LINC01614, biomarker, malignancies, oncogene, pan-cancer
2018 (1). The therapeutic strategies for treating numerous types of cancer are usually ineffective, primarily due to the lack of specific diagnostic and prognostic markers (2). Diagnostic biomarkers could be used for screening or early detection of cancer, while prognostic biomarkers could be used to predict the likelihood of recurrence or progression in patients with cancer (2). Accordingly, it is necessary to identify novel biomarkers for potential clinical applications (3-5).

Long non-coding RNAs (IncRNAs) have crucial roles in the progression of cancer (6-10). Certain lncRNAs are involved in the modulation of neoplastic proliferation, invasion and metastasis. In addition, several studies have indicated that lncRNAs may serve as potential cancer-specific biomarkers (2,11-13).

The gene encoding long intergenic non-protein-coding RNA 1614 (LINC01614), also known as lung cancer-associated lncRNA 4, is located on chromosome 2 q35 in between two exons and was originally identified as being upregulated in lung cancer (14). In addition, Liu et al (15) reported that LINC01614 knockdown inhibits cell proliferation through the microRNA (miR)-217/forkhead box (FOX)P1 axis in lung adenocarcinoma. Another study indicated that LINC01614 is highly expressed in breast cancer tissue and associated with poor prognosis based on the data from The Cancer Genome Atlas (TCGA) (16). Increasing evidence has indicated that LINC01614 may act as an oncogene and serve as a biomarker in several types of cancer (15-18). However, beyond the limited information provided by these studies, the role of LINC01614 in malignant tumors has remained elusive.

In the present study, a comprehensive pan-cancer analysis of the data available from public databases was performed to determine the correlation between LINC01614 expression and the prognoses of various malignancies. In addition, bioinformatics analyses were performed to identify the physiological functions of this lncRNA to clarify its potential role as an oncogene. Furthermore, the expression levels in paired normal and tumor tissue specimens from patients with non-small cell lung cancer (NSCLC) or colon cancer were assessed to validate the results.

\section{Materials and methods}

Malignancy-associated microarray and RNA sequencing data in the Gene Expression Omnibus (GEO) and TCGA databases. Microarray and RNA sequencing data on LINC01614 
expression were systematically searched in the GEO database on 26th June 2019 (http://www.ncbi.nlm.nih.gov/geo/). The search terms were as follows: (neoplasm OR cancer OR tumor OR carcinoma) AND (lncRNA OR long non-coding RNA). The inclusion criteria were as follows: i) Expression data for LINC01614 were provided or it was possible to calculate them for each sample; ii) samples in each dataset included cancer tissues and normal tissues or cancer tissues with prognostic information; iii) the number of samples in each dataset was $>20$; and iv) the species in each dataset was Homo sapiens. Samples based on cell lines were excluded. Gene expression matrices were downloaded from the GEO database and extracted using $\mathrm{R}$ and associated packages.

Datasets including RNA sequencing and clinical data for 24 types of cancer from TCGA were downloaded and extracted usingtheTCGAbiolinkspackage(https://gdc-portal.nci.nih.gov/). RNA sequencing (RNA-seq) expression data were normalized using RNA-seq by Expectation-Maximization (19).

Comparison of LINC01614 expression in normal and tumor tissues. LINC01614 expression in normal tissues was examined from the RNA-seq data obtained from the Genotype-Tissue Expression (GTEx) (https://www.gtexportal.org/home/) database, comprising $\sim 11,600$ samples from 53 tissue types. The pre-processed data were downloaded from the portal and converted to the units of transcripts per million (TPM) for comparison of relative expression levels among different tissues. The RNA-seq data from TCGA for libraries with sufficient malignant and adjacent normal tissues were also used to evaluate the expression of LINC01614 in malignancies. The RNA-seq expression matrices of these datasets were normalized by $\log 2(\mathrm{x}+1)$ transformation. The relative expression levels were statistically compared between the tumor tissues and adjacent normal tissues using unpaired Student's t-tests.

Receiver operating characteristic (ROC) and summary ROC (SROC) curve analysis. Datasets with sufficient malignant and adjacent normal tissues were used to evaluate the diagnostic value of LINC01614 in malignancies. ROC curve analysis was performed to evaluate the area under the curve (AUC) with 95\% CIs, sensitivities and specificities for each dataset. SROC curve analysis was performed using Stata 14.2 (StataCorp LLC) to demonstrate pooled sensitivity, pooled specificity and obtain the pooled AUC. $\mathrm{I}^{2}$ and Q tests were used to assess the heterogeneity of this meta-analysis. Meta-regression analysis was performed to determine the potential cause of heterogeneity. Deeks' funnel plots were used to evaluate the potential publication bias of the SROC analysis.

Survival analysis and comprehensive meta-analysis. The above-mentioned GEO data with survival information and TCGA data were used to assess the prognostic value of LINC01614. Samples in each dataset were divided into two groups according to the cut-off value determined from ROC curves (20). Univariate Cox regression analysis was performed to obtain hazard ratios (HRs) with $95 \%$ CIs for overall survival (OS). Pooled HRs and 95\% CIs were combined to assess the association between LINC01614 expression and prognosis in various malignancies. If the $95 \% \mathrm{CI}$ of the combined HR did not overlap with 1 , the results were considered significant. If
$\mathrm{I}^{2}>50 \%$ or $\mathrm{P} \leq 0.05$, the heterogeneity was considered significant and a random-effects model was chosen; if not, a fixed-effects model was used. A sensitivity analysis was also performed to assess the stability of the combined results and a subgroup analysis was performed to detect possible sources of heterogeneity. Begg's test and the trim fill method were used to assess the potential publication bias.

Tissue samples and clinical data collection. For validation of the RNA-seq data, 74 and 78 tumor tissue samples (alongside matched adjacent normal tissue samples) from patients with non-small cell lung cancer (NSCLC) or colon cancer, respectively, who underwent resection at Beijing Tongren Hospital (Beijing, China) between October 2016 and March 2018, were analyzed. The tissue samples were immersed in RNA later (Ambion) and stored at $-80^{\circ} \mathrm{C}$ until use. The clinicopathological characteristics of each patient were obtained from their medical records. The study was approved by the ethics committee of Beijing Tongren Hospital (Beijing, China).

$R N A$ extraction and $R T-q P C R$. Total RNA was extracted from the tissue samples with TRIzol reagent (Invitrogen; Thermo Fisher Scientific, Inc.) according to the manufacturer's protocol. The RNA integrity was detected using $1 \%$ agarose gel electrophoresis and a spectrophotometer was used to measure its concentration and purity. The $28 \mathrm{~S}: 18 \mathrm{~S}$ ribosomal RNA ratio of RNA samples was required to be 2:1; the A260/A280 ratios were 1.8-2.2 and the absorbance at $260 \mathrm{~nm}$ (A260)/A230 ratios were required to be $>1.7$ for the RNA samples to be considered qualified. RNA was used to synthesize cDNA using the SuperScript III Reverse Transcriptase kit (Thermo Fisher Scientific, Inc.) according to the manufacturer's protocol; the mixture included $1 \mu \mathrm{g}$ total RNA, $1 \mu \mathrm{l}$ oligo(dT) $)_{20}, 1 \mu \mathrm{ldNTP}$ Mix, $1 \mu 1$ 0.1M DTT, $1 \mu 1$ RNaseOUT, $1 \mu 1$ SuperScript $^{\text {TM }}$ III $\mathrm{RT}$ and enzyme-free water in a $20 \mu \mathrm{l}$ reaction volume. The reaction conditions used were $50^{\circ} \mathrm{C}$ for $60 \mathrm{~min}$ and $70^{\circ} \mathrm{C}$ for $15 \mathrm{~min}$. Reverse transcription-quantitative (RT-q)PCR was performed using the SYBR-Green Mix (Takara Bio, Inc.) on an ABI 7500 system (Applied Biosystems; Thermo Fisher Scientific, Inc.). The following thermocycling conditions were used for qPCR: Pre-denaturation at $94^{\circ} \mathrm{C}$ for $2 \mathrm{~min}, 40$ cycles of denaturation at $94^{\circ} \mathrm{C}$ for $5 \mathrm{sec}$ and annealing at $60^{\circ} \mathrm{C}$ for $30 \mathrm{sec}$, and a final extension step at $72^{\circ} \mathrm{C}$ for $10 \mathrm{~min}$. The expression levels were normalized to those of GAPDH mRNA (16) using the $2^{-\Delta \Delta \mathrm{Cq}}$ method (21), where $\Delta \mathrm{Cq}=(\mathrm{Cq}$ of the target-Cq GAPDH) and $\Delta \Delta \mathrm{Cq}=\Delta \mathrm{Cq}$ tumor tissues $-\Delta \mathrm{Cq}$ adjacent non-tumor tissues. Each sample was analyzed in triplicate. The sequences of the primers used in the study were as follows: LINC01614 forward, 5'-GGGACTTCAGACACGGAGAA-3' and reverse, 5'-GGACACAGACCCTAGCACTT-3'; collagen XI $\alpha 1$ chain (COL11A1) forward, 5'-TAACATCGCTGACGGGAAGTG-3' and reverse, 5'-CCGTGATTCCATTGGTATCAACA-3'; secreted protein acidic and cysteine rich (SPARC) forward, 5'-CCCATTGGCGAGTTTGAGAAG-3' and reverse, 5'-CAA GGCCCGATGTAGTCCA-3'; periostin (POSTN) forward, 5'-CAACGGGCAAATACTGGAAAC-3' and reverse, 5'-TCT CGCGGAATATGTGAATCG-3'; COL5A2 forward, 5'-ACA GGGTTTACAAGGACAGCA-3' and reverse, 5'-GGTCCA GGATCACCAGGTT-3'; fibroblast activation protein $\alpha$ (FAP) forward, 5'-TCAGCTATGATGCCATTTCG-3' and reverse, 


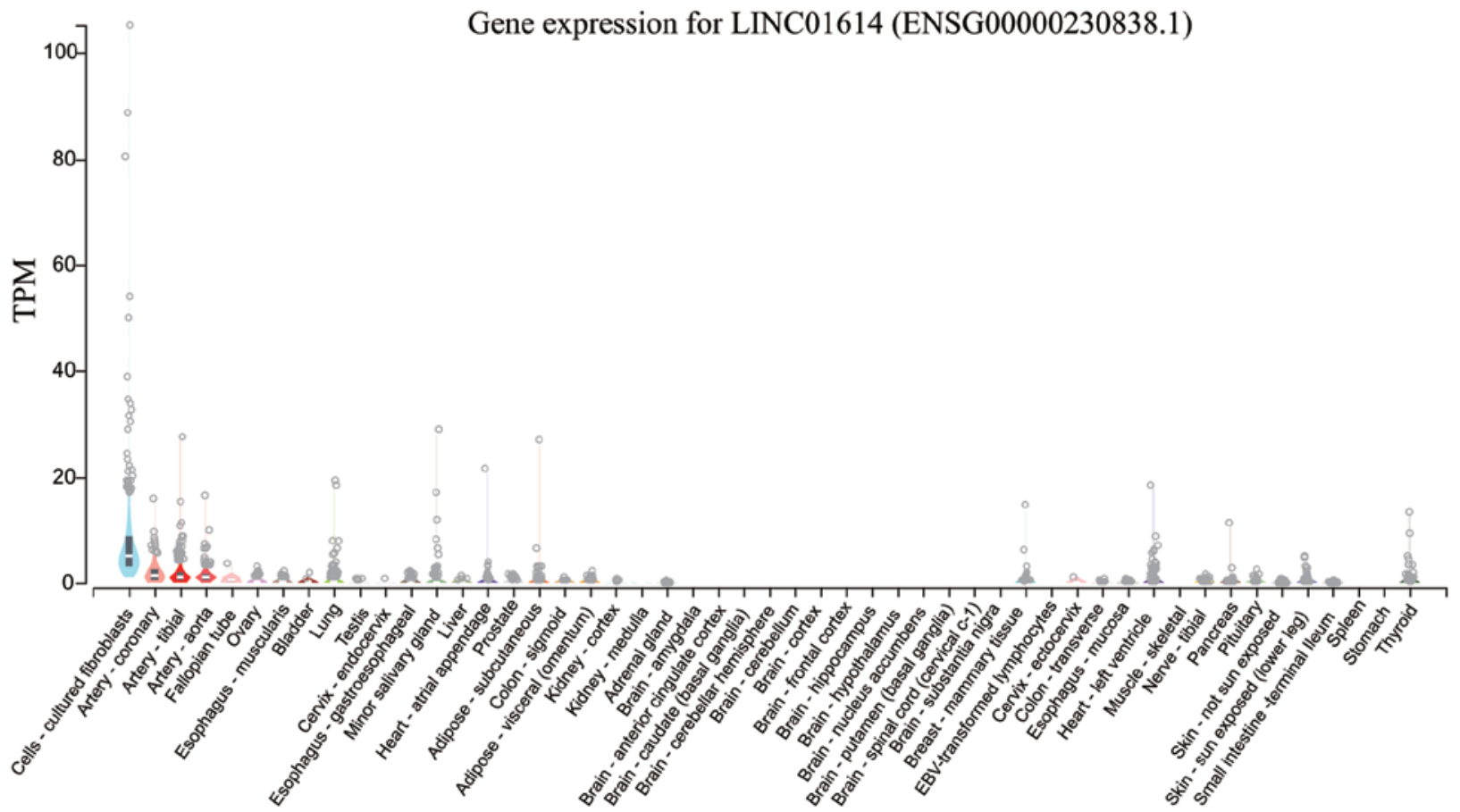

Figure 1. Expression of LINC01614 in normal tissues. Violin plots were used to express the TPM data of LINC01614 in each tissue. The colors indicate the different tissues. The outliers are marked as circles. LINC01614, long intergenic non-coding RNA 1614; TPM, transcripts per million.

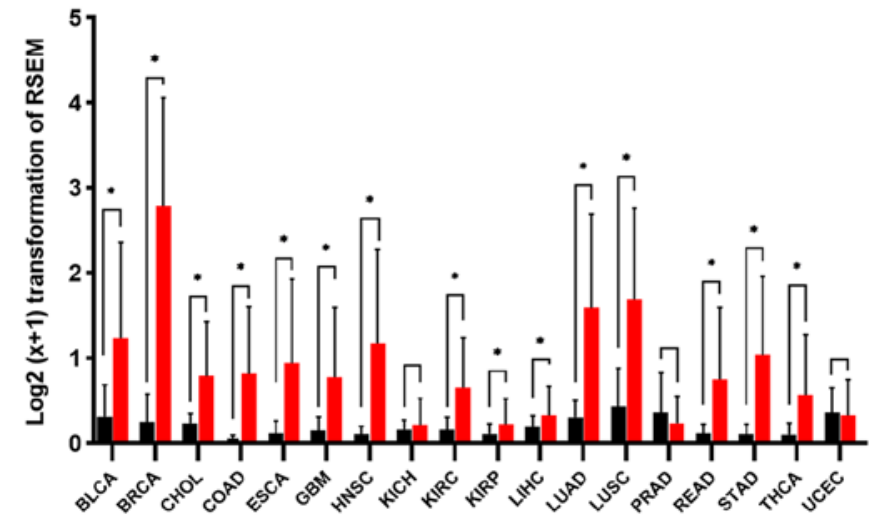

Figure 2. Expression of long intergenic non-coding RNA 1614 in malignancies based on The Cancer Genome Atlas data. Significance was determined using an unpaired Student's t-test. Values are expressed as the mean \pm SD. ${ }^{*} \mathrm{P}<0.05$. RSEM, RNA-sequencing Expectation-Maximization; BLCA, bladder urothelial carcinoma; BRCA, breast invasive carcinoma; CHOL, cholangiocarcinoma; COAD, colon adenocarcinoma; ESCA, esophageal carcinoma; GBM, glioblastoma multiforme; HNSC, head and neck squamous cell carcinoma; KICH, kidney chromophobe; KIRC, kidney renal clear cell carcinoma; KIRP, kidney renal papillary cell carcinoma; LIHC, liver hepatocellular carcinoma; LUAD, lung adenocarcinoma; LUSC, lung squamous cell carcinoma; PRAD, prostate adenocarcinoma; READ, rectum adenocarcinoma; STAD, stomach adenocarcinoma; THCA, thyroid carcinoma; UCEC, uterine corpus endometrial carcinoma.

5'-CCTCCCACTTGCCACTTGTA-3'; and GAPDH forward, 5'-CAACTCCCTCAAGATTGTCAGCAA-3' and reverse, 5'-GGCATGGACTGTGGTCATGA-3'.

Bioinformatics analysis of LINC01614. To investigate the molecular pathways associated with the upregulation of LINC01614 in malignancies, gene set enrichment analysis (GSEA) was performed using the GSEA software 3.0 (http:// software.broadinstitute.org/gsea/msigdb/index.jsp) (22). Annotated gene sets 'c2.cp.kegg.v6.2.symbols.gmt' and 'h.all. v6.2.symbols.gmt' were downloaded from the Molecular Signatures Database (23). The normalized enrichment score was determined by the analysis of 1,000 permutations. A gene set was considered significantly enriched when the false discovery rate was $<0.25$. In addition, Pearson's correlation coefficient was determined to identify the genes co-expressed with LINC01614. Genes with Pearson's r-values $>0.3$ and $\mathrm{P}<0.01$ were considered significant.

Statistical analysis. Microarray and RNA-seq data were analyzed using $\mathrm{R}$, version 3.5.2, and associated packages. An unpaired Student's t-test, the $\chi^{2}$ test, ROC curve analysis and univariate Cox regression analysis were performed using SPSS Statistics v22.0 (IBM Corp.). Diagnostic and prognostic meta-analyses were conducted using Stata 14.2 (StataCorp LLC). The measurement data were expressed as the mean \pm SD. Student's t-test and the $\chi^{2}$ test were used to analyze the significance of differences between groups. $\mathrm{P}<0.05$ was considered to indicate a statistically significant difference.

\section{Results}

LINC01614 expression is high in most tumor tissues but low in most normal tissues. To determine the general distribution of LINC01614 in normal tissues, its expression was first examined across 53 different tissues in the GTEx database. As presented in Fig. 1, the median expression levels of LINC01614 in different tissue types ranged from a TPM of 0 (vagina) to 5.213 (cultured fibroblasts). Except for the cultured fibroblasts (median TPM=5.213), coronary artery 


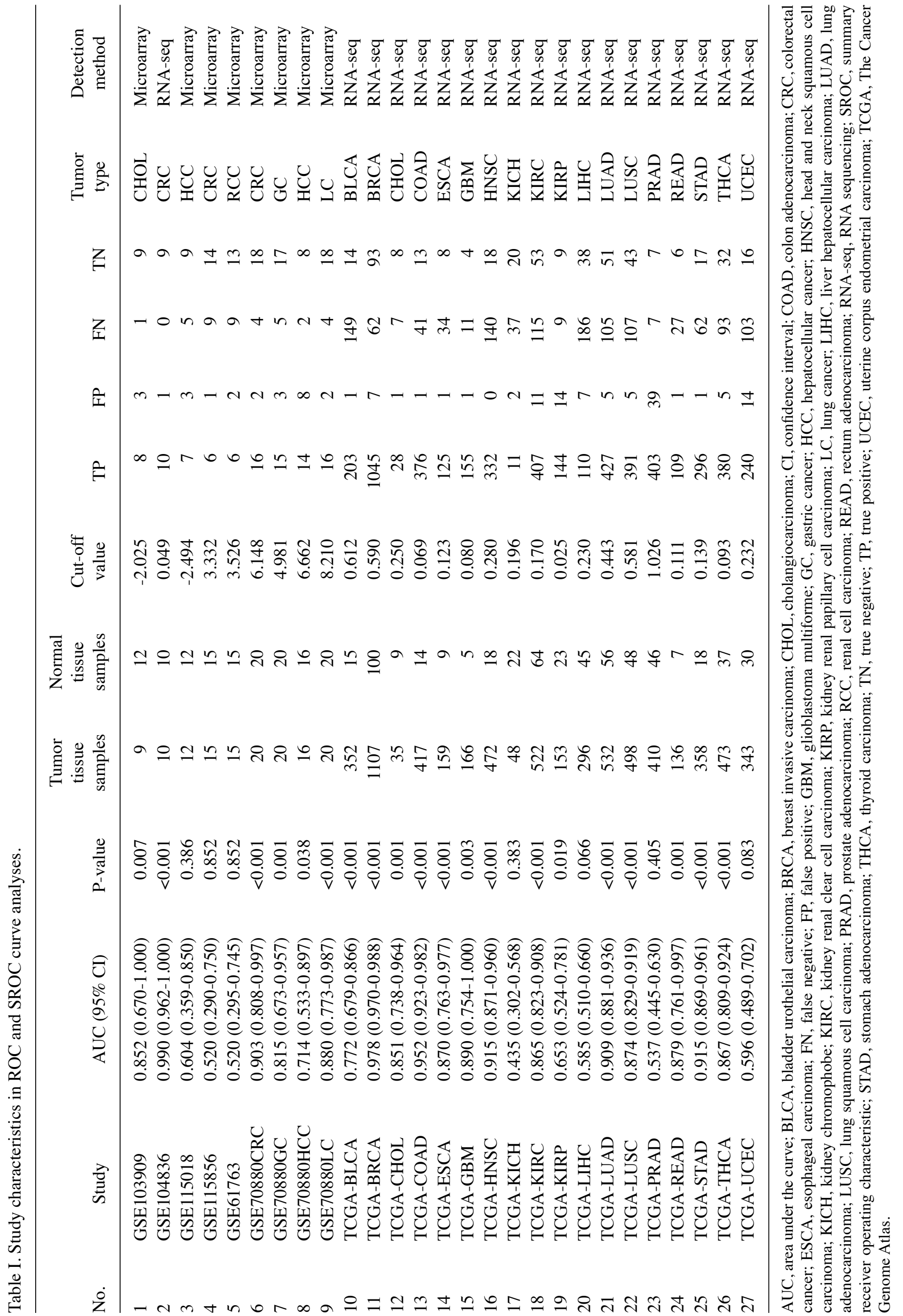


A

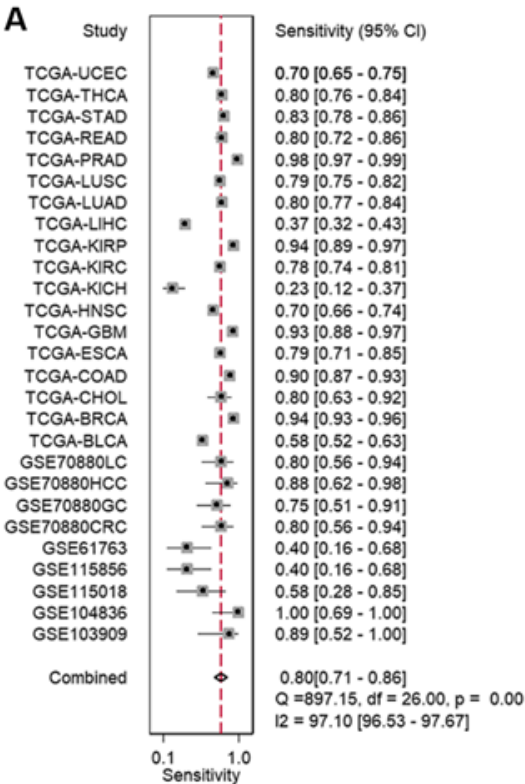

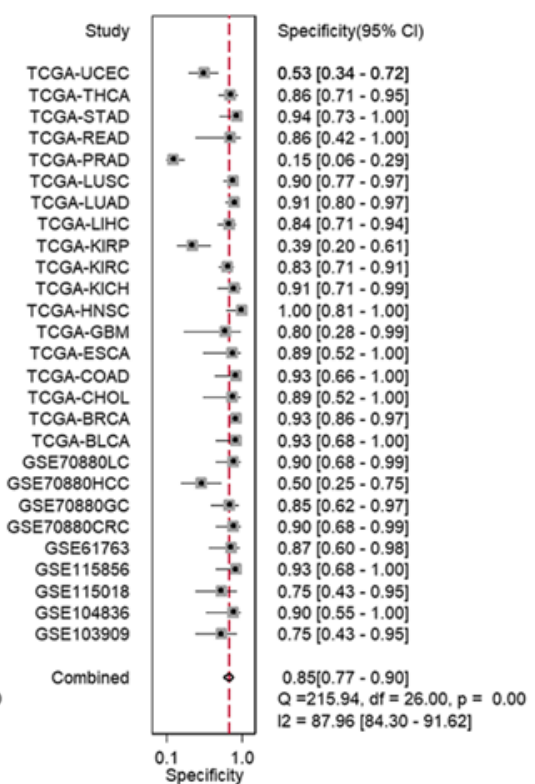

$\mathrm{SROC}$ with prediction and confidence contours

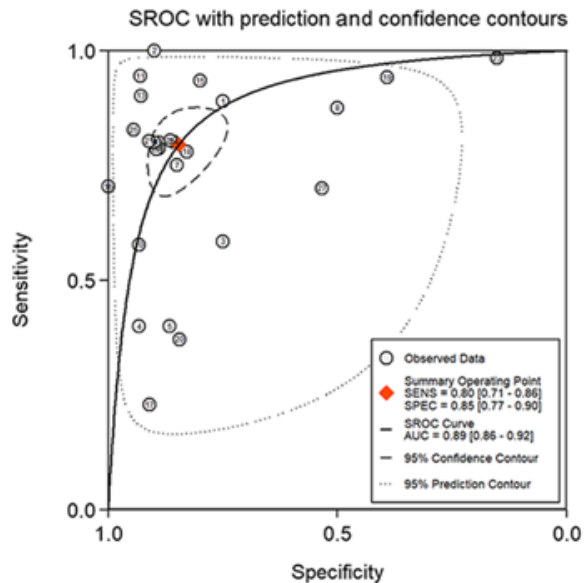

B Univariable meta-regression and subgroup analyses

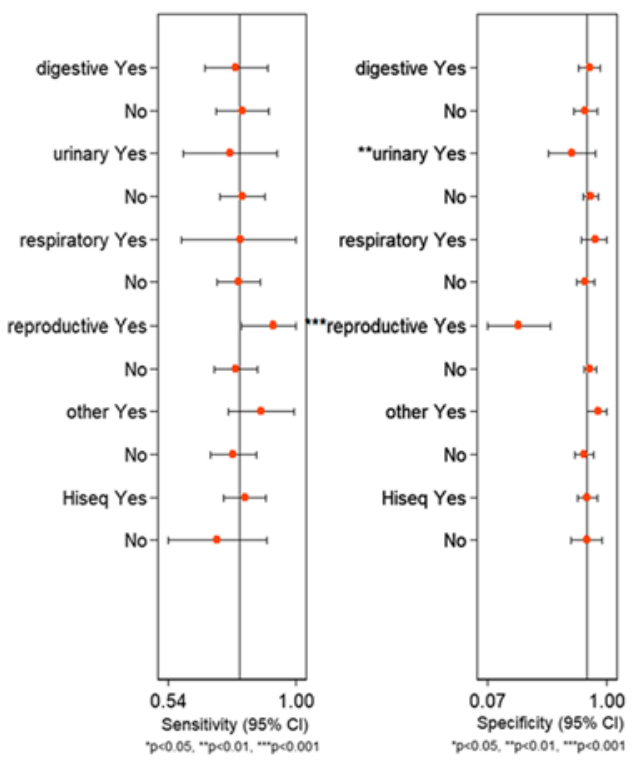

D

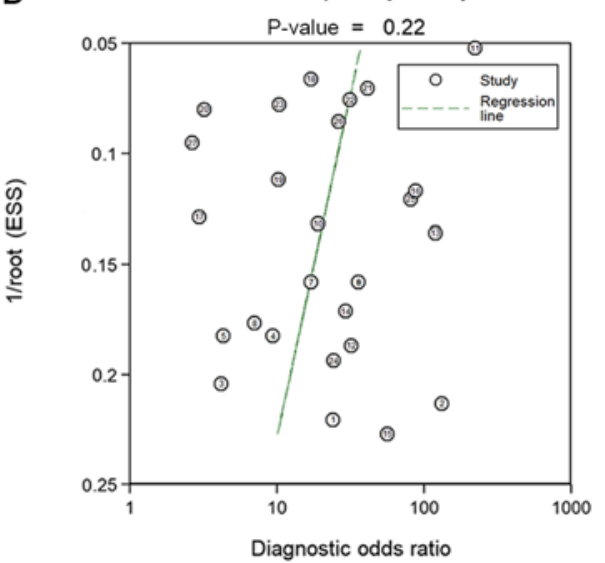

Figure 3. (A) Combined sensitivity and specificity of long intergenic non-coding RNA 1614 for the diagnosis of malignancies. (B) Meta-regression analysis indicated that reproductive system cancers $(\mathrm{P}<0.001)$ and urinary system cancers $(\mathrm{P}=0.01)$ may be the major causes of heterogeneity. (C) SROC curve and (D) Deeks' funnel plot analyses. The numbers in the circles represent the number of each study in Table I. The overall diagnostic efficiency was summarized by the regression curve. SROC, summary receiver operating characteristic; AUC, area under curve; TCGA, The Cancer Genome Atlas; BLCA, bladder urothelial carcinoma; BRCA, breast invasive carcinoma; CHOL, cholangiocarcinoma; COAD, colon adenocarcinoma; CRC, colorectal cancer; ESCA, esophageal carcinoma; GBM, glioblastoma multiforme; GC, gastric cancer; HCC, hepatocellular cancer; HNSC, head and neck squamous cell carcinoma; KICH, kidney chromophobe; KIRC, kidney renal clear cell carcinoma; KIRP, kidney renal papillary cell carcinoma; LC, lung cancer; LIHC, liver hepatocellular carcinoma; LUAD, lung adenocarcinoma; LUSC, lung squamous cell carcinoma; PRAD, prostate adenocarcinoma; READ, rectum adenocarcinoma; STAD, stomach adenocarcinoma; THCA, thyroid carcinoma; UCEC, uterine corpus endometrial carcinoma; SENS, sensitivity; SPEC, specificity; ESS, explained sum of square.

(median TPM=1.496), tibial artery (median TPM=1.012) and aorta (median TPM=1.009), all of which exhibited relatively high levels of LINC01614, most of the other tissues had low levels (median TPM <0.5).

After excluding the datasets that had an insufficient amount of normal tissue samples, 18 types of cancers with 7,043 samples from TCGA were selected to evaluate the expression patterns of LINC01614 in cancers. As presented in Fig. 2, most tumors (16 of them) had higher LINC01614 levels than the normal tissues. Unpaired Student's t-tests indicated that LINC01614 was significantly upregulated in 15 types of malignant tissues, including bladder carcinoma (BLCA), breast invasive carcinoma (BRCA), cholangiocarcinoma, colon adenocarcinoma, esophageal carcinoma, glioblastoma multiforme, head and neck squamous cell carcinoma (HNSC), kidney renal clear cell carcinoma (KIRC), kidney renal papillary cell carcinoma, liver hepatocellular carcinoma, lung adenocarcinoma (LUAD), lung squamous cell carcinoma (LUSC), rectum adenocarcinoma (READ), stomach adenocarcinoma (STAD) and thyroid carcinoma $(\mathrm{P}<0.05)$. Furthermore, the expression levels of LINC01614 were relatively low in malignant prostate adenocarcinoma (PRAD) and uterine corpus endometrial carcinoma (UCEC) tissues but not significantly different from the levels in the adjacent normal tissues $(\mathrm{P}>0.05)$. Other normal tissue types had low levels of LINC01614 when it was highly expressed in the adjacent tumor tissues.

LINC01614 may serve as a diagnostic predictor based on GEO and TCGA databases. In total, 590 datasets were identified 


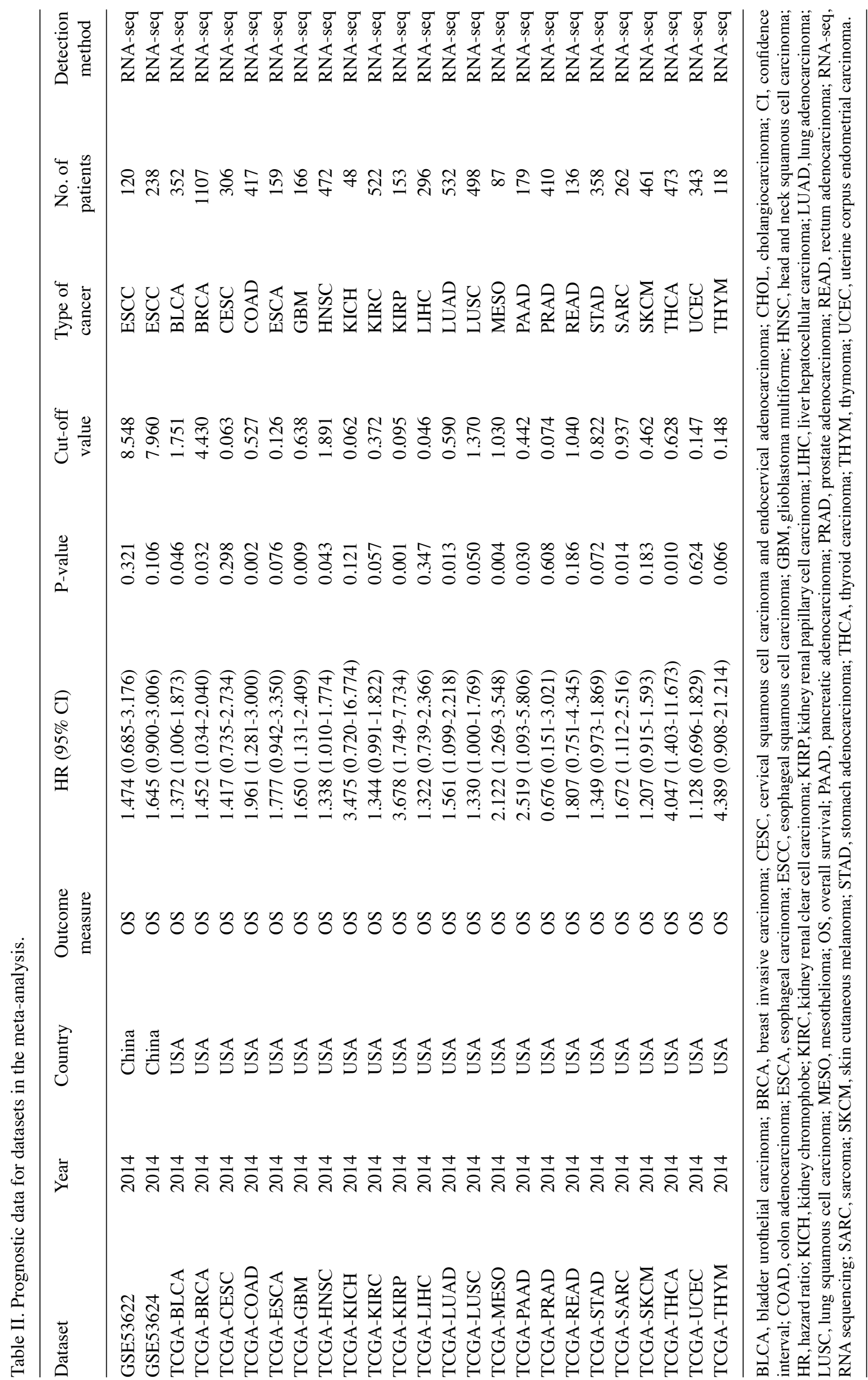



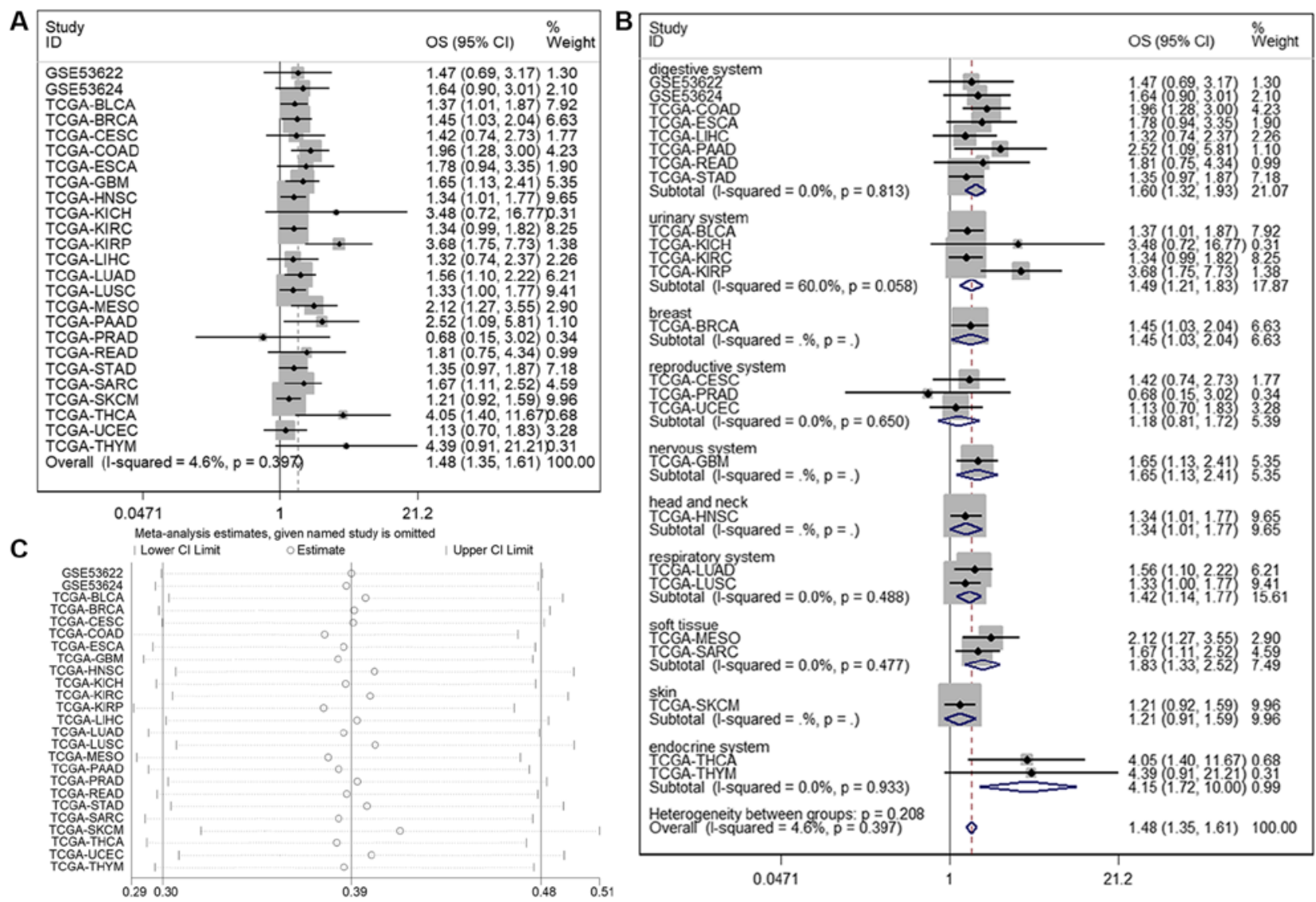

Figure 4. (A) Forest plots for the association between LINC01614 expression and OS. (B) Forest plots of subgroup analysis for OS. (C) Sensitivity analysis of datasets to evaluate the associations between LINC01614 expression and OS. LINC01614, long intergenic non-coding RNA 1614; OS, overall survival; TCGA, The Cancer Genome Atlas; BLCA, bladder urothelial carcinoma; BRCA, breast invasive carcinoma; CESC, cervical squamous cell carcinoma and endocervical adenocarcinoma; CHOL, cholangiocarcinoma; COAD, colon adenocarcinoma; ESCA, esophageal carcinoma; GBM, glioblastoma multiforme; HNSC, head and neck squamous cell carcinoma; KICH, kidney chromophobe; KIRC, kidney renal clear cell carcinoma; KIRP, kidney renal papillary cell carcinoma; LIHC, liver hepatocellular carcinoma; LUAD, lung adenocarcinoma; LUSC, lung squamous cell carcinoma; MESO, mesothelioma; PAAD, pancreatic adenocarcinoma; PRAD, prostate adenocarcinoma; READ, rectum adenocarcinoma; SARC, sarcoma; SKCM, skin cutaneous melanoma; STAD, stomach adenocarcinoma; THCA, thyroid carcinoma; THYM, thymoma; UCEC, uterine corpus endometrial carcinoma.

during the primary search, among which 39 were downloaded later from the GEO database after relevant assessments and evaluations. Eventually, eight datasets were included in the analysis (GSE70880, GSE115856, GSE61763, GSE103909, GSE104836, GSE115018, GSE53622 and GSE53624) after screening in accordance with the inclusion criteria. Finally, after excluding the datasets that had an insufficient amount of normal tissue samples, 24 datasets, including 7,320 patients, from GEO and TCGA databases were used to assess the diagnostic predictive ability of LINC01614. The AUCs, sensitivities and specificities of these datasets are presented in Table I. The results revealed that $\mathrm{P}<0.05$ for the ROC curve in 20/27 cohorts, which indicated that LINC01614 was a suitable diagnostic marker for numerous human malignancies. The combined sensitivities and specificities of LINC01614 for the diagnosis of malignancies are presented in Fig. 3. Subsequently, the SROC curve analysis was performed to determine the diagnostic value of LINC01614 in malignancies. The combined sensitivity, specificity and AUC of the SROC curve were 0.80 (95\% CI, 0.71-0.86), 0.85 (95\% CI, 0.77-0.90) and 0.89 (95\% CI, 0.86-0.92), respectively (Fig. 3A and C). The combined results indicated that LINC01614 was suitable to diagnose human malignancies, even though there was a marked heterogeneity among the results. Deeks' funnel plots indicated that there was no significant publication bias in the SROC curve analysis $(\mathrm{P}=0.22$; Fig. 3D). Thus, a meta-regression analysis was performed to determine the cause of heterogeneity. The results indicated that cancers of the reproductive $(\mathrm{P}<0.001)$ and urinary system $(\mathrm{P}=0.01)$ may be a major cause of heterogeneity (Fig. 3B). Of note, UCEC, $\mathrm{KICH}$ and PRAD, which exhibited no significant differences in LINC0164 expression between tumor and normal tissues, are all cancers of the reproductive or urinary system. Therefore, these results indicated that except for certain cancers of these systems, LINC01614 may be a potential diagnostic biomarker for the majority of malignancies.

Prognostic value of LINC01614 for OS in malignancies. In total, 23 TCGA and two GEO datasets (8,213 patients) were included to analyze the association between LINC01614 expression and survival in human cancers. The HR and 95\% CI of each dataset were obtained by univariate Cox regression model analysis (Table II). As shown in Fig. 4, the pooled HR from all the datasets indicated a significant association between high LINC01614 expression and poor OS in various malignant tumors $(\mathrm{HR}=1.479 ; 95 \% \mathrm{CI}, 1.355-1.614$; 
Table III. Subgroup analysis of overall survival based on primary tumors of different organ systems in the meta-analysis.

\begin{tabular}{lccccrr}
\hline Subgroup & No. of studies & No. of patients & Pooled HR (95\% CI) & PHet & $I^{2}(\%)$ & P-value \\
\hline Digestive system & 8 & 1903 & $1.598(1.321,1.933)$ & 0.813 & 0.0 & $<0.001$ \\
Urinary system & 4 & 1075 & $1.491(1.212,1.833)$ & 0.058 & 60.0 & $<0.001$ \\
Reproductive system & 3 & 649 & $1.177(0.808,1.716)$ & 0.650 & 0.0 & 0.396 \\
Respiratory system & 2 & 1030 & $1.417(1.136,1.769)$ & 0.488 & 0.0 & 0.002 \\
Soft tissue & 2 & 349 & $1.833(1.332,2.524)$ & 0.477 & 0.0 & $<0.001$ \\
Endocrine system & 2 & 591 & $4.151(1.723,9.998)$ & 0.933 & 0.0 & 0.002 \\
Breast & 1 & 1107 & $1.452(1.034,2.039)$ & - & - & 0.031 \\
Nervous system & 1 & 166 & $1.650(1.131,2.408)$ & - & - & 0.009 \\
Head and neck & 1 & 472 & $1.338(1.010,1.773)$ & - & - & 0.043 \\
Skin & 1 & 461 & $1.207(0.915,1.593)$ & - & - & 0.183 \\
\end{tabular}

The datasets in each subgroup are shown in Fig. 4B. HR, hazard ratio; PHet, P-value for heterogeneity.
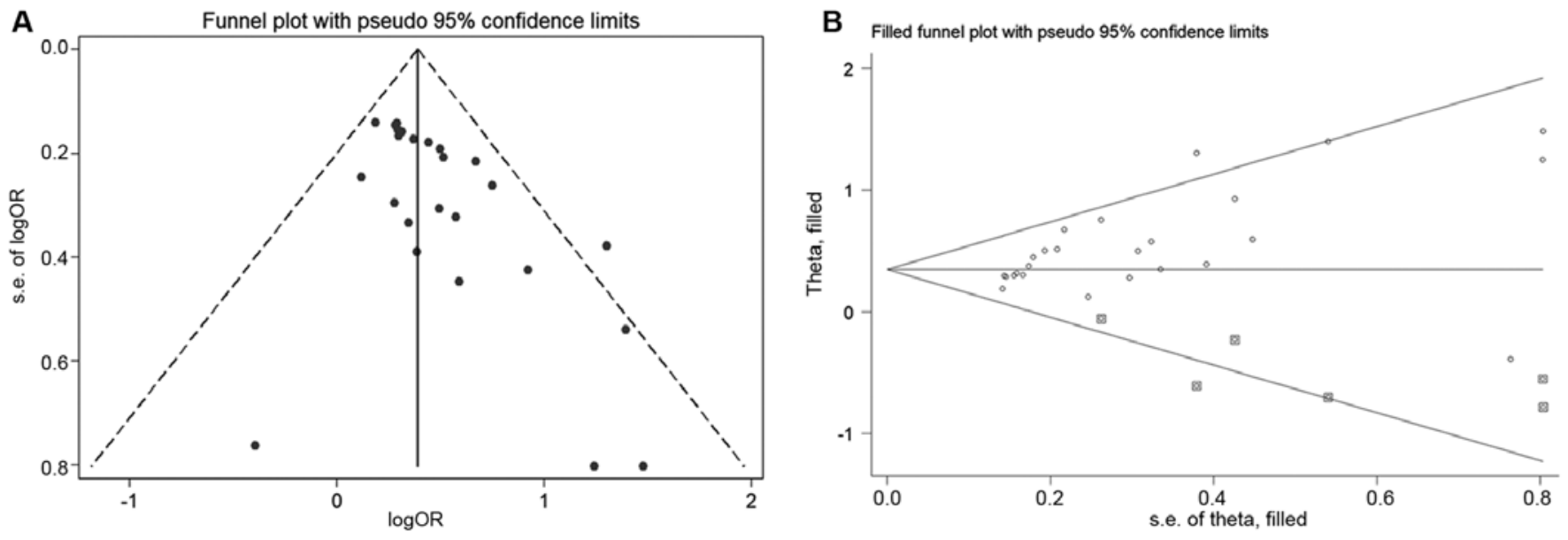

Figure 5. (A) Begg's funnel plot for publication bias analysis of overall survival. (B) After adjusting for filling six assumed studies, the adjusted HR and 95\% CI exhibited no significant changes compared with the initial HR and 95\% CI. The assumed studies were indicated by small boxes. OR, odds ratio; s.e., standard error.

$\mathrm{P}<0.001)$. To assess the stability of the combined results, a sensitivity analysis of OS was performed. The results of the sensitivity analysis suggested that no individual study changed the combined results; thus, the pooled results were reliable (Fig. 4C). Publication bias was assessed by Begg's test and the results suggested that there was a considerable publication bias in this meta-analysis $(\mathrm{P}=0.001$; Fig. $5 \mathrm{~A})$. To further verify the reliability of the results, a trim and fill analysis was performed to eliminate the impact of publication bias by establishing symmetry assumptions (24). As indicated by the filled funnel plots in Fig. 5B, six assumed studies were filled to eliminate the publication bias. After adjusting for filling assumed studies, the adjusted HR and 95\% CI (1.414 and 1.299-1.539, respectively) was not significantly different compared with the initial HR and 95\% CI (1.479 and 1.355-1.614, respectively), confirming that the results were reliable.

To assess potential heterogeneity, a subgroup analysis of OS was performed based on different organ systems. The results suggested that upregulation of LINC01614 was significantly associated with poor OS in malignancies from most organ systems, including the digestive $(\mathrm{P}<0.001)$, urinary $(\mathrm{P}<0.001)$, nervous $(\mathrm{P}=0.009)$, respiratory $(\mathrm{P}=0.002)$ and endocrine $(\mathrm{P}=0.002)$ systems, in addition to the malignancies involving head and neck $(\mathrm{P}=0.043)$, breast $(\mathrm{P}=0.031)$ and soft tissue $(\mathrm{P}<0.001)$, whereas there was no association with cancers from the reproductive system $(\mathrm{P}=0.396)$ or skin $(\mathrm{P}=0.183)$ (Table III and Fig. 4B). Of note, only cancers of the urinary system had significant heterogeneity within the subgroup, suggesting that LINC01614 has distinct roles in these four different urinary cancers. LINC01614 may be an oncogene and prognostic marker for BLCA or KIRP, but not for KICH or KIRC.

Bioinformatics analysis identified LINC01614-associated signaling pathways in human cancers. To identify the possible LINC01614-associated signaling pathways in human cancers, GSEA was performed on eight TCGA datasets with a weight of prognostic analyses of $>5$, including BLCA, BRCA, HNSC, KIRC, LUAD, LUSC, skin cutaneous melanoma and STAD. To eliminate the effects of tissue specificity, the intersection between these GSEA results from different datasets was 

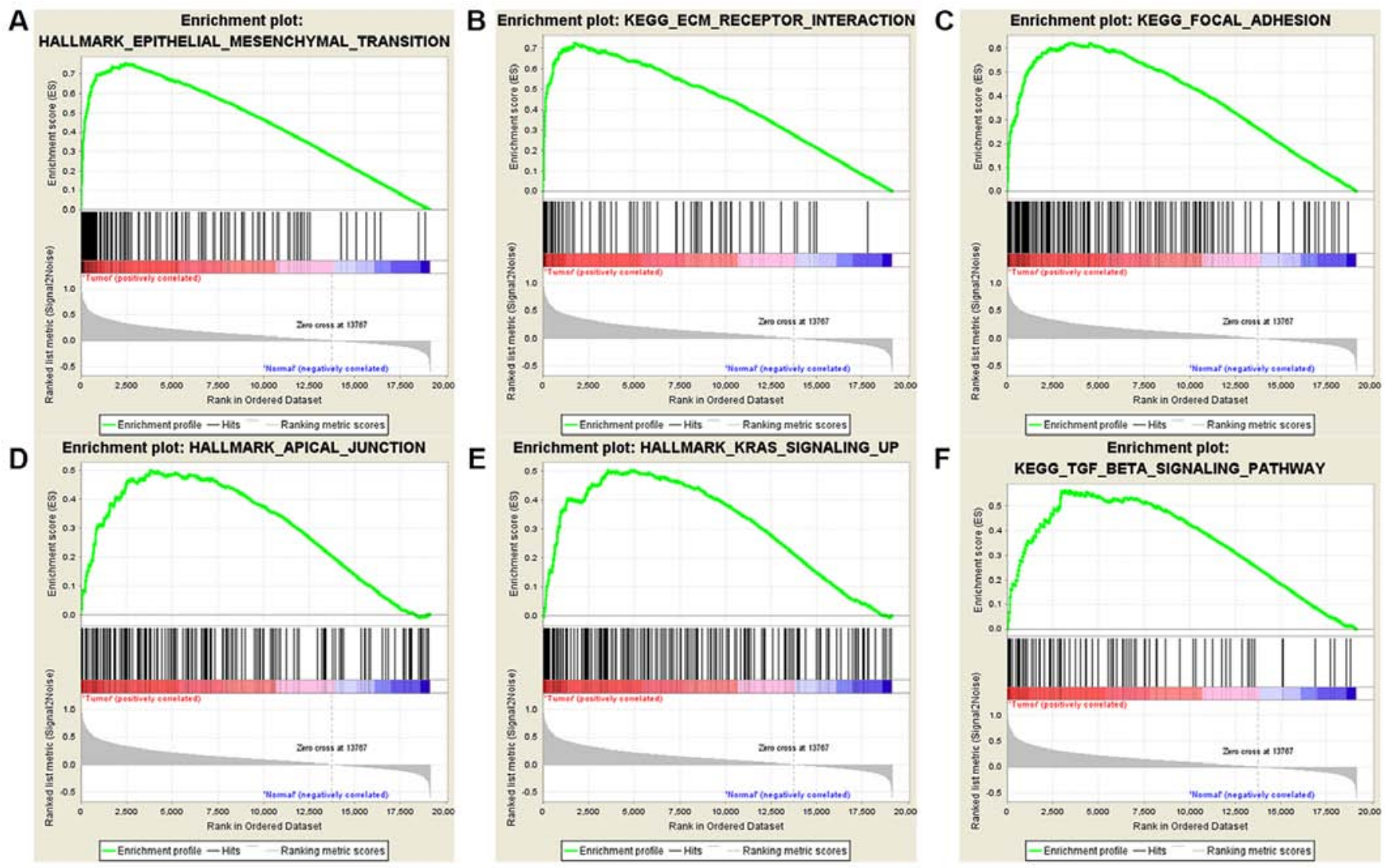

Figure 6. Gene set enrichment analysis of signaling pathways based on The Cancer Genome Atlas - head and neck squamous cell carcinoma datasets Enrichment plots for (A) the hallmark epithelial-mesenchymal transition, (B) KEGG-ECM receptor interaction; (C) KEGG focal adhesion, (D) the hallmark apical junction, (E) the hallmark KRAS signaling upregulated and (F) KEGG TGF- $\beta$ signaling pathway. KEGG, Kyoto Encyclopedia of Genes and Genomes; ECM, extracellular matrix; TGF, transforming growth factor.

used. As illustrated in Figs. 6, 7 and S1-7, and Tables SI-IX, the GSEA enrichment plots indicated that LINC01614 expression was highly associated with the epithelial-mesenchymal transition (EMT), extracellular matrix (ECM) receptor interaction, focal adhesion, gap junction, adherens junction and cell adhesion molecules. Furthermore, LINC01614 expression was associated with angiogenesis, apoptosis, KRAS signaling, transforming growth factor- $\beta$ (TGF- $\beta$ ) signaling, tumor necrosis factor- $\alpha$ (TNF- $\alpha$ ) signaling, mitogen-activated protein kinase (MAPK) signaling, Wnt signaling and various cancer-associated signaling pathways.

Pearson's correlation coefficient was then used to evaluate the genes that were co-expressed with LINC01614 in these TCGA datasets. To obtain the most significantly co-expressed genes, the overlapping results of eight different datasets (Pearson's $\mathrm{r}>0.3, \mathrm{P}<0.01$ ) were obtained. As illustrated in Fig. 8, 36 genes were significantly co-expressed with LINC01614 and 32 of these genes were associated with EMT. Based on the biological roles of LINC01614 in cancers, it was concluded that LINC01614 mainly has an important role in EMT and associated signaling pathways in malignancies.

RT-qPCR validation of LINC01614 and co-expressed gene levels in cancer tissues. To confirm the reliability and validity of the public RNA-seq data, the expression profiles of LINC01614 and five significantly co-expressed genes, including COL11A1, FAP, POSTN, SPARC and COL5A2, were analyzed using
RT-qPCR in paired samples from 74 patients with NSCLC and 78 patients with colon cancer. The results indicated that LINC01614 was expressed at a significantly higher level in NSCLC and colon cancer tumor tissues than their respective adjacent normal tissues (Fig. 9A and B). The diagnostic accuracies of LINC01614 for NSCLC and colon cancer were investigated by ROC analyses, which indicated a sensitivity, specificity and AUC of $0.743,0.905$ and 0.868 for NSCLC (cutoff value $=1.287$ ) and of $0.795,0.923$ and 0.876 for colon cancer (cutoff value $=0.625$ ), respectively (Fig. 9C and D). Further analysis of the association between LINC01614 and clinicopathological characteristics in the cohorts of the present study suggested that higher LINC01614 expression was significantly associated with tumor size, lymph node metastasis and TNM stage in patients with NSCLC, and with lymph node metastasis and TNM stage in patients with colon cancer (Tables IV and V). These results indicated that the expression of LINC01614 was higher in NSCLC and colon cancer tissues and may function as an oncogene in these cancers.

Furthermore, Pearson's correlation analysis between LINC01614 and co-expressed genes, including COL11 A1, FAP, POSTN, SPARC and COL5A2 in NSCLC and colon cancer tissues, was performed. The results indicated that the expression of LINC01614 was markedly positively correlated with the levels of COL11A1, FAP, POSTN, SPARC, and COL5A2 in NSCLC and colon cancer (Figs. 10 and 11). These results were in line with those of the comprehensive bioinformatics 


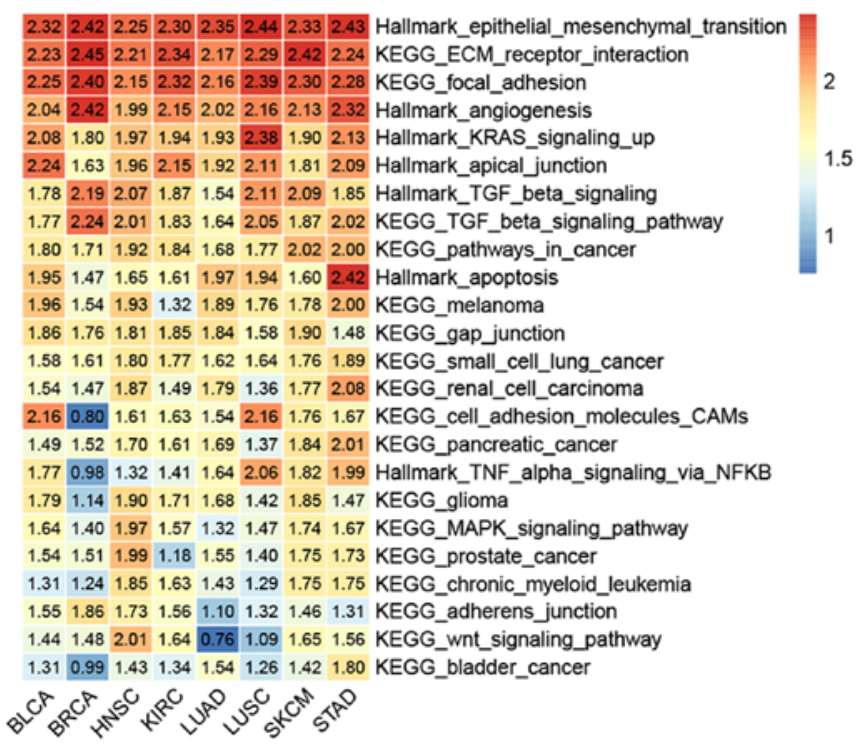

Figure 7. Heatmap of gene set enrichment analysis data, indicating the association of long intergenic non-coding RNA 1614 expression with various signaling pathways in all the eight datasets. KEGG, Kyoto Encyclopedia of Genes and Genomes; ECM, extracellular matrix; TGF, transforming growth factor; TNF, tumor necrosis factor; MAPK, mitogen-activated protein kinase; CAMs, cell adhesin molecules; BLCA, bladder urothelial carcinoma; BRCA, breast invasive carcinoma; HNSC, head and neck squamous cell carcinoma; KIRC, kidney renal clear cell carcinoma; LUAD, lung adenocarcinoma; LUSC, lung squamous cell carcinoma; SKCM, skin cutaneous melanoma; STAD, stomach adenocarcinoma.

analyses described above, demonstrating the credibility of the RNA-seq data.

\section{Discussion}

lncRNAs act as crucial regulators of almost all aspects of physiological and pathological processes (25-28). Accumulating evidence indicates that lncRNAs contribute to the carcinogenesis and progression of various cancers (6-10). LINC01614 was initially reported as an upregulated lncRNA in lung cancer (14). Further studies demonstrated that LINC01614 promotes the progression of lung adenocarcinoma by competing with miR-217 and preventing the miR-217/FOXP1 interaction (15). Dysregulation of LINC01614 expression has also been reported in breast carcinoma and indicated to be associated with poor prognosis (16). Emerging evidence suggests that LINC01614 acts as an oncogene and may be a potential biomarker in malignancies. However, its expression and clinical implication in a full spectrum of cancers have remained to be determined. To the best of our knowledge, the present study was the first to comprehensively assess the role of LINC01614 in cancer. Owing to the continuous development of high-throughput sequencing, abundant high-throughput data from human cancers have been collected in public databases. The public data in these databases have increasingly crucial roles in the study of cancers (29). Based on large-sample high-throughput data from public databases, the present study aimed to investigate the diagnostic and prognostic value of LINC01614 in malignancies.

In the present study, comprehensive analyses across 53 normal tissue types and 24 tumor types were performed, including data mining, differential expression analyses, ROC and SROC analyses, survival analyses, meta-analyses and bioinformatics analyses. Overall, the results suggested that the majority of normal tissues have a low expression of LINC01614. Statistical analysis (t-tests) further revealed that LINC01614 was significantly upregulated in 15 of the 18 types of malignant tissues compared with the expression levels in their normal tissues. Furthermore, ROC and SROC curve analyses indicated that LINC01614 is of suitable diagnostic value in various malignancies. However, meta-regression analysis revealed that cancers of the reproductive system and urinary system contributed to the significant heterogeneity of the SROC curve analysis. In line with this result, UCEC, KICH and PRAD, which are cancers of the reproductive or urinary system, exhibited no significant alterations between tumor and normal tissues. Therefore, except for several cancers of these systems, LINC01614 was significantly upregulated in malignant tissue samples and may be a potential diagnostic biomarker for the majority of malignancies, including cancers of the digestive, respiratory, nervous and endocrine systems, in addition to the malignancies of the breast and head and neck.

In addition, a meta-analysis was performed to determine the prognostic predictive capacity of LINC01614 in cancers. The pooled results indicated that upregulation of LINC01614 was significantly associated with poor OS in malignant tumors from most organ systems, including the digestive, urinary, nervous, respiratory and endocrine systems, as well as of the head and neck, breast and soft tissue with the exception of cancers of the reproductive system or skin. In addition, only cancers of the urinary system caused significant heterogeneity in this subgroup.

Overall, abnormal expression of LINC01614 and its associations with clinical outcome were highly cancer-dependent. LINC01614 had no reliable diagnostic or prognostic value in some cancers of the reproductive or urinary system. However, for cancers of the digestive system, respiratory system, breast, head and neck, nervous system and endocrine system, LINC01614 was proved to be an oncogene and credible biomarker with diagnostic and prognostic value.

The results of the RT-qPCR validation also indicated that the expression of LINC01614 was higher in NSCLC and colon cancer tissues compared with the expression levels in their respective adjacent normal tissues; high LINC01614 expression was associated with lymph node metastasis and tumor stage, further supporting its role as an oncogene in these cancers. In addition, 9 of the 78 colon cancer patients had liver metastasis. In this cohort, there were more patients with liver metastasis in the high-expression group (7 of 38) than in the low-expression group ( 2 of 38), but the difference was not statistically significant $(\mathrm{P}=0.069)$. This insignificant difference may be due to the limited sample size. LINC01614 is able to promote liver and lymph node metastases in colon cancer. These validation results demonstrated that the comprehensive analyses were reliable.

At present, the molecular mechanisms of LINC01614 in cancers remain to be fully elucidated. Therefore, bioinformatics analyses were performed to discover the inherent molecular mechanisms of LINC01614. The results indicated that LINC01614 may have important effects on the progression of cancers by modulating several cellular biology 


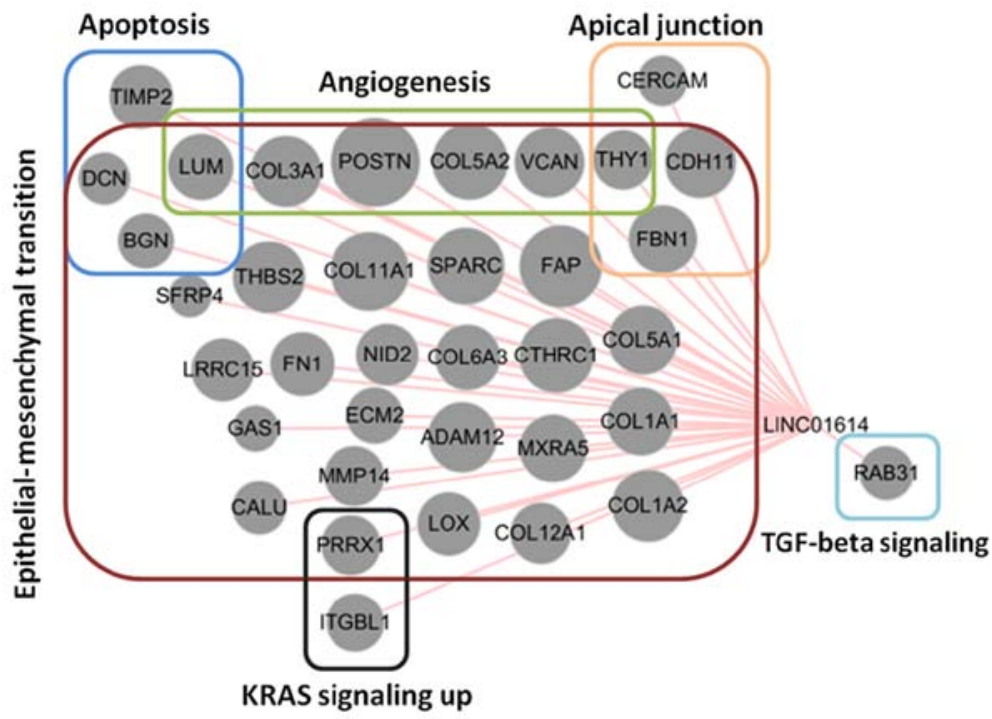

Figure 8. Association of genes co-expressed with LINC01614 during epithelial to mesenchymal transition based on the overlapping results from the eight The Cancer Genome Atlas datasets. The sizes of the nodes represent the mean values of Pearson's correlation coefficients among the eight datasets. LINC01614, long intergenic non-coding RNA 1614; TGF, transforming growth factor; ADAM12, ADAM metallopeptidase domain 12; BGN, biglycan; CALU, calumenin; CDH11, cadherin 11; CERCAM, cerebral endothelial cell adhesion molecule; COL11A1, collagen XI $\alpha 1$ chain; COL12A1, collagen type XII $\alpha 1$ chain; COL1A1, collagen type I $\alpha 1$ chain; COL1A2, collagen type I $\alpha 2$ chain; COL3A1, collagen type III $\alpha 1$ chain; COL4A1, collagen type IV $\alpha 1$ chain; COL5A1, collagen type $\mathrm{V} \alpha 1$ chain; COL5A2, collagen type $\mathrm{V} \alpha 2$ chain; COL5A3, collagen type $\mathrm{V} \alpha 3$ chain; COL6A2, collagen type VI $\alpha 2$ chain; COL6A3, collagen type VI $\alpha 3$ chain; COL8A2, collagen type VIII $\alpha 2$ chain; COMP, cartilage oligomeric matrix protein; CTHRC1, collagen triple helix repeat containing 1; DCN, decorin; DPYSL3, dihydropyrimidinase like 3; ECM2, extracellular matrix protein 2; FAP, fibroblast activation protein $\alpha$; FBN1, fibrillin 1; FERMT2, fermitin family member 2; FN1, fibronectin 1; GAS1, growth arrest specific 1; GFPT2, glutamine-fructose-6-phosphate transaminase 2; GREM1, gremlin 1; HTRA1, HtrA serine peptidase 1; ITGBL1, integrin subunit $\beta$ like 1; LGALS1, galectin 1; LOX, lysyl oxidase; LRP1, LDL receptor related protein 1; LRRC15, leucine rich repeat containing 15; LUM, lumican; MMP, matrix metallopeptidase; MXRA5, matrix remodeling associated 5; NID2, nidogen 2; NTM, neurotrimin; PDGFRB, platelet-derived growth factor receptor $\beta$; POSTN, periostin; PRRX1, paired related homeobox 1; RAB31, member RAS oncogene family; SFRP4, secreted frizzled related protein 4; SPARC, secreted protein acidic and cysteine rich; THBS2, thrombospondin 2; THY1, Thy-1 cell surface antigen; TIMP2, TIMP metallopeptidase inhibitor 2; TPM4, tropomyosin 4; VCAN, versican.

A
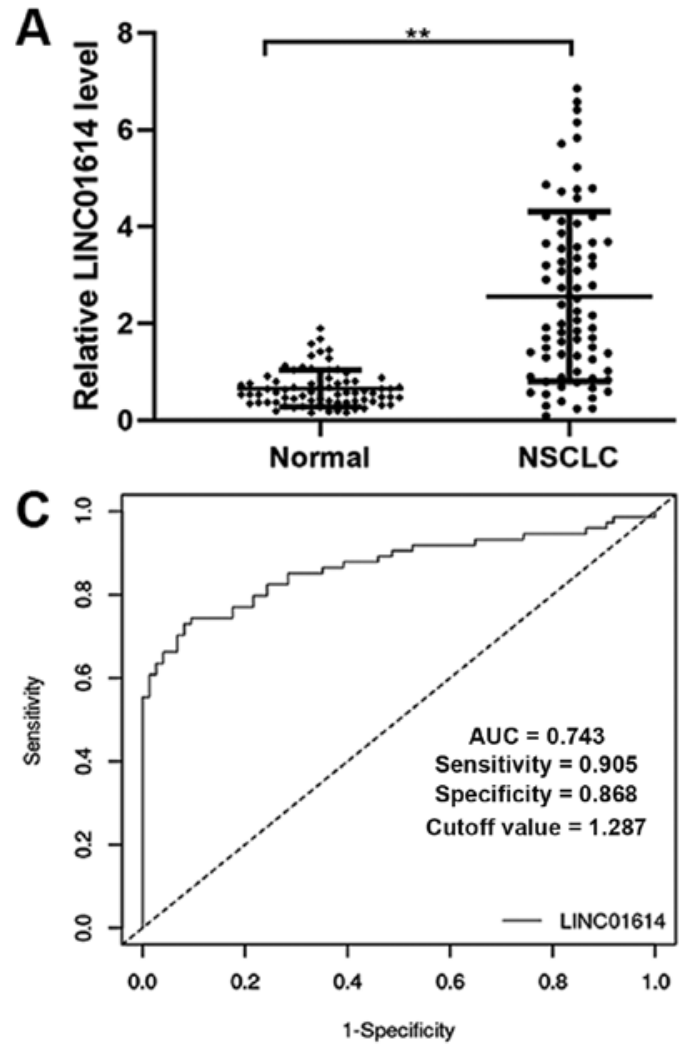

B
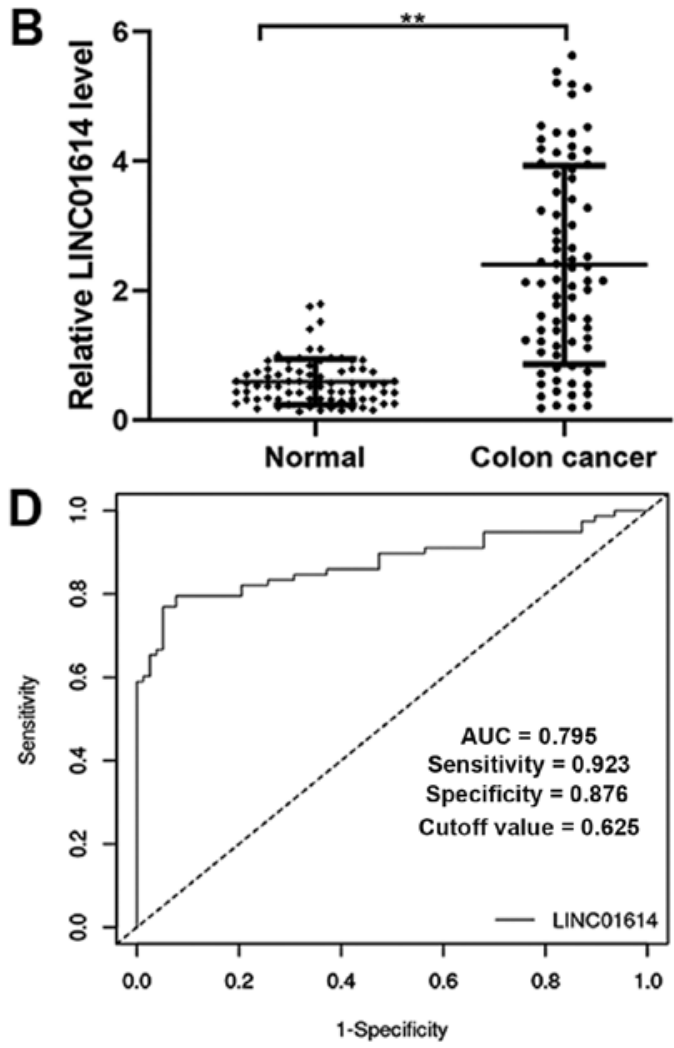

Figure 9. Expression of LINC01614 in (A) NSCLC and (B) colon cancer determined by RT-qPCR. Receiver operating characteristic curve analyses based on the RT-qPCR results for (C) NSCLC and (D) colon cancer. ${ }^{* *} \mathrm{P}<0.01$. LINC01614, long intergenic non-coding RNA 1614; NSCLC, non-small cell lung cancer; RT-qPCR, reverse transcription-quantitative PCR. 
Table IV. Association between LINC01614 and clinicopathological characteristics of patients with non-small cell lung cancer $(n=74)$.

LINC01614

\begin{tabular}{|c|c|c|c|c|}
\hline \multirow{2}{*}{ Clinicopathologic parameters } & \multirow[b]{2}{*}{ Total (n) } & & & \\
\hline & & Low $(n=37)$ & High $(n=37)$ & P-value \\
\hline Sex & & & & 0.104 \\
\hline Male & 37 & 15 & 22 & \\
\hline Female & 37 & 22 & 15 & \\
\hline Age (years) & & & & 0.235 \\
\hline$\leq 60$ & 14 & 5 & 9 & \\
\hline$>60$ & 60 & 32 & 28 & \\
\hline Size of tumor $(\mathrm{cm})$ & & & & 0.047 \\
\hline$\leq 3$ & 24 & 16 & 8 & \\
\hline$>3$ & 50 & 21 & 29 & \\
\hline Lymph node metastasis & & & & 0.032 \\
\hline No & 45 & 27 & 18 & \\
\hline $\mathrm{N} 1-3$ & 29 & 10 & 19 & \\
\hline Degree of differentiation & & & & 0.344 \\
\hline Moderate-well & 44 & 24 & 20 & \\
\hline Poor & 30 & 13 & 17 & \\
\hline Histological type & & & & 0.642 \\
\hline Adenocarcinoma & 36 & 19 & 17 & \\
\hline Squamous carcinoma & 38 & 18 & 20 & \\
\hline Smoking & & & & 0.483 \\
\hline Yes & 41 & 19 & 22 & \\
\hline No & 33 & 18 & 15 & \\
\hline TNM stage & & & & 0.016 \\
\hline I & 35 & 23 & 12 & \\
\hline II & 25 & 7 & 18 & \\
\hline III/IV & 14 & 7 & 7 & \\
\hline
\end{tabular}

The cutoff value for classification into high and low LINC01614 expression was based on the median of the relative expression levels of LINC01614. LINC01614, long intergenic non-coding RNA 1614; TNM, tumor-node-metastasis.

processes, including the EMT, ECM receptor interaction, focal adhesion, gap junction, angiogenesis, apoptosis, KRAS signaling, TGF- $\beta$ signaling, TNF- $\alpha$ signaling and various cancer-associated signaling pathways. Furthermore, most of the genes identified to be co-expressed with LINC01614 were associated with EMT in human cancers. Positive correlations between LINC01614 and five co-expressed genes, including COL11A1, FAP, POSTN, SPARC and COL5A2, were identified in NSCLC and colon cancer tissues. Relevant studies have revealed that COL11A1 and COL5A2 are important EMT mediators and are involved in tumorigenesis and metastasis of several cancers (30-33). According to previous studies, FAP derived from cancer-associated fibroblasts was able to be induced by EMT through Wnt/ $\beta$-catenin signaling $(34,35)$. Overexpression of POSTN was able to induce EMT through the MAPK/ERK pathway $(36,37)$. Furthermore, SPARC was able to increase the migration and invasion of cancer cells by promoting EMT through the phosphorylated (p)-FAK/p-ERK pathway $(38,39)$. These results suggested that LINC01614 may be involved in EMT and associated signaling pathways to influence the progression of human cancers.

During EMT, cells lose their epithelial properties, generating migratory mesenchymal cell types with highly invasive characteristics (40-42). In addition, cancer cells undergoing EMT exhibit increased robustness including inhibition of apoptosis and senescence, and acquisition of immunosuppression and drug resistance $(43,44)$. Metastasis is the major cause of mortality in patients with cancer, and cancer cells that obtain invasive characteristics through EMT have a crucial role during metastasis (45-47). The present bioinformatics analyses revealed that a high level of LINC01614 may be involved in EMT and associated signaling pathways to influence cancer progression and metastasis. In line with this, RT-qPCR validation also suggested that high LINC01614 expression was associated with lymph node metastasis and tumor staging in colon cancer and NSCLC. This observation may be attributed 
Table V. Association between LINC01614 and clinicopathological characteristics in patients with colorectal cancer ( $\mathrm{n}=78$ ).

\begin{tabular}{|c|c|c|c|c|}
\hline \multirow[b]{2}{*}{ Clinicopathologic parameter } & \multirow[b]{2}{*}{ Total (n) } & \multicolumn{3}{|c|}{ LINC01614 } \\
\hline & & Low $(n=39)$ & High $(n=39)$ & P-value \\
\hline Sex & & & & 0.174 \\
\hline Male & 40 & 17 & 23 & \\
\hline Female & 38 & 22 & 16 & \\
\hline Age (years) & & & & 0.645 \\
\hline$\leq 60$ & 32 & 15 & 17 & \\
\hline$>60$ & 46 & 24 & 22 & \\
\hline $\mathrm{T}$ classification & & & & 0.098 \\
\hline T1-2 & 11 & 8 & 3 & \\
\hline T3-4 & 67 & 31 & 36 & \\
\hline Lymph node metastasis & & & & 0.006 \\
\hline No & 44 & 28 & 16 & \\
\hline N1-2 & 34 & 11 & 23 & \\
\hline Distant metastasis & & & & 0.069 \\
\hline M0 & 69 & 37 & 32 & \\
\hline M1 & 9 & 2 & 7 & \\
\hline Degree of differentiation & & & & 0.131 \\
\hline Moderate-well & 56 & 31 & 25 & \\
\hline Poor & 22 & 8 & 14 & \\
\hline Location & & & & 0.460 \\
\hline Right & 32 & 15 & 17 & \\
\hline Transverse & 11 & 4 & 7 & \\
\hline Left & 10 & 7 & 3 & \\
\hline Sigmoid colon & 25 & 13 & 12 & \\
\hline TNM stage & & & & 0.022 \\
\hline $\mathrm{I} / \mathrm{II}$ & 44 & 27 & 17 & \\
\hline $\mathrm{III} / \mathrm{IV}$ & 34 & 12 & 22 & \\
\hline
\end{tabular}

The cutoff value for classification into high and low LINC01614 expression was based on the median of the relative expression levels of LINC01614. LINC01614, long intergenic non-coding RNA 1614; TNM, tumor-node-metastasis.

to the influence of LINC01614 on the EMT. The positive correlation between LINC01614 and EMT-associated genes, including COL11A1, FAP, POSTN, SPARC and COL5A2, also confirmed this biological process. Further studies are required to validate the molecular mechanisms whereby LINC01614 modulates EMT in malignancies.

There are numerous strengths to the present study. First, it was a pan-cancer comprehensive analysis, including 53 normal tissue types and 32 cancer datasets with data from 9,091 patients, representing a complete dataset available for LINC01614 in human cancers. These large-sample data from different databases were able to enhance the reliability of this comprehensive analysis. Furthermore, the reliability and validity of the results of the comprehensive analysis were confirmed with a molecular biology technique with clinical samples obtained at our hospital. Finally, the results of the bioinformatics analyses were validated in eight cancer datasets. The rigorous evaluation criteria further supported the reliability and repeatability of the results.
However, the study also has certain limitations. First, significant heterogeneity existed in certain analyses in this study. The heterogeneity was derived from cancers originating from certain specific tissues that were validated by meta-regression and subgroup analyses. Furthermore, the diagnostic value of LINC01614 was identified in fresh solid tissues, but the early diagnostic value requires to be validated using the blood samples of cancer patients. In addition, due to the limited sample size, the association between LINC01614 and certain clinicopathological characteristics may be false-negative, including liver metastasis in colon cancer, and this requires further investigation with larger sample sizes and multicenter research. Finally, the molecular mechanisms of LINC01614 were not identified by this approach. Accordingly, further targeted studies are required to verify these results.

In summary, LINC01614 emerged as an oncogene in most malignancies, and its abnormal expression and clinical 

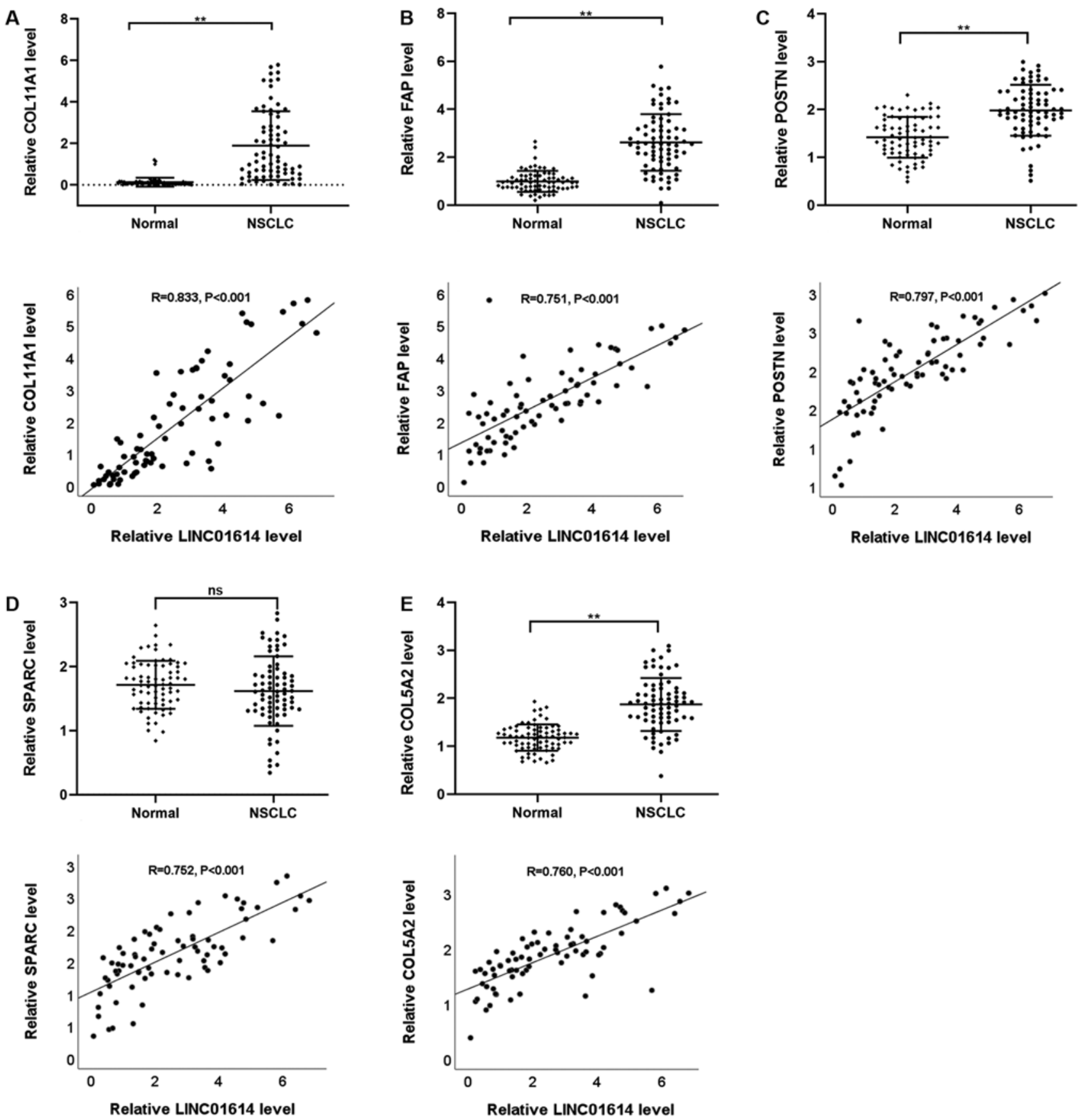

Figure 10. Expression and Pearson's correlation analysis between LINC01614 and (A) COL11A1, (B) FAP, (C) POSTN, (D) SPARC and (E) COL5A2 in NSCLC. ${ }^{* *} \mathrm{P}<0.01$. ns, not significant; LINC01614, long intergenic non-coding RNA 1614; NSCLC, non-small cell lung cancer; COL11A1, collagen XI $\alpha 1$ chain; FAP, fibroblast activation protein $\alpha$; POSTN, periostin; SPARC, secreted protein acidic and cysteine rich.

outcome associations were highly cancer-dependent. For cancers of the digestive system, respiratory system, breast, head and neck, nervous system and endocrine system, LINC01614 is a credible biomarker with diagnostic and prognostic value. However, for certain cancers of the reproductive and urinary system, LINC01614 had no significant clinical association. Furthermore, bioinformatics analysis indicated that LINC01614 may promote the progression and metastasis of malignancies by modulating the EMT in most malignancies. For future clinical applications, comprehensive studies are required to assess the detailed molecular mechanisms underlying the oncogenic role of LINC01614.

\section{Acknowledgements}

Not applicable.

\section{Funding}

The present study was supported by the Beijing Municipal Education Commission (grant no. KZ201910025033), Beijing Municipal Administration of Hospitals Clinical Medicine Development of Special Funding (grant no. ZYLX201814) and National Natural Science Foundation of China (grant no. 81502493). 

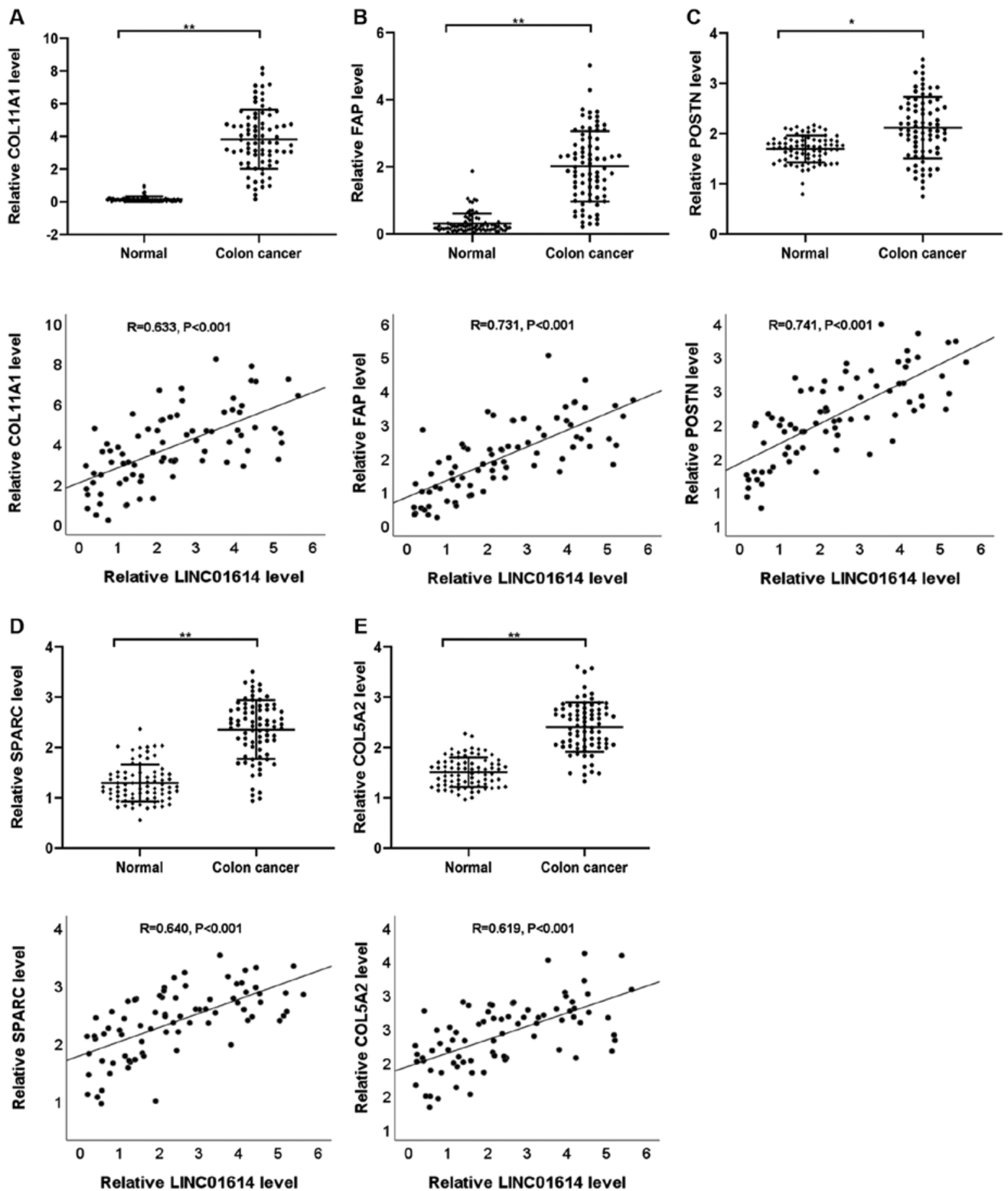

Figure 11. Expression and Pearson's correlation analysis between LINC01614 and COL11A1 (A) FAP (B) POSTN (C) SPARC (D) and COL5A2 (E) in colon cancer. ${ }^{*} \mathrm{P}<0.05 ;{ }^{* *} \mathrm{P}<0.01$. LINC01614, long intergenic non-coding RNA 1614; COL11A1, collagen XI $\alpha 1$ chain; FAP, fibroblast activation protein $\alpha$; POSTN, periostin; SPARC, secreted protein acidic and cysteine rich.

\section{Availability of data and materials}

The datasets used and/or analyzed during the current study are available from the corresponding author on reasonable request.

\section{Authors' contributions}

Design of the study: HL. Preparation of the manuscript: DW. Conception of the study: HZ and XF. Editing of the manuscript:
DW and XF. Preparation of figures: XZ and HZ. Data analysis: DW and HZ. Statistics: DW and DC. All authors contributed toward the revision of the manuscript and agree to be accountable for all aspects of the work. All authors read and approved the final manuscript.

\section{Ethics approval and consent to participate}

The present study was approved by the ethics committee of Beijing Tongren Hospital (Beijing, China; 
approval no. 20180212). All patients provided written informed consent and agreed to participate in the study.

\section{Patient consent for publication}

Not applicable.

\section{Competing interests}

The authors declare that they have no competing interests.

\section{References}

1. Bray F, Ferlay J, Soerjomataram I, Siegel RL, Torre LA and Jemal A: Global cancer statistics 2018: GLOBOCAN estimates of incidence and mortality worldwide for 36 cancers in 185 countries. CA Cancer J Clin 6: 394-424, 2018.

2. Ng L, Poon RT and Pang R: Biomarkers for predicting future metastasis of human gastrointestinal tumors. Cell Mol Life Sci 70: 3631-3656, 2013.

3. Fidler MM, Gupta S, Soerjomataram I, Ferlay J, Steliarova-Foucher E and Bray F: Cancer incidence and mortality among young adults aged 20-39 years worldwide in 2012: A population-based study. Lancet Oncol 18: 1579-1589, 2017.

4. Kim JL, Cho KH, Park EC and Cho WH: A single measure of cancer burden combining incidence with mortality rates for worldwide application. Asian Pac J Cancer Prev 15: 433-439, 2014.

5. Qin L, Chen C, Chen L, Xue R, Ou-Yang M, Zhou C, Zhao S, $\mathrm{He} \mathrm{Z}$, Xia Y, He J, et al: Worldwide malaria incidence and cancer mortality are inversely associated. Infect Agent Cancer 12: 14, 2017.

6. Dhamija S and Diederichs S: From junk to master regulators of invasion: lncRNA functions in migration, EMT and metastasis. Int J Cancer 139: 269-280, 2016.

7. Bhan A and Mandal SS: LncRNA HOTAIR: A master regulator of chromatin dynamics and cancer. Biochim Biophys Acta 1856: 151-164, 2015.

8. Yang G, Lu X and Yuan L: LncRNA: A link between RNA and cancer. Biochim Biophys Acta 1839: 1097-1109, 2014.

9. Lin $\mathrm{C}$ and Yang L: Long noncoding RNA in cancer: Wiring signaling circuitry. Trends Cell Biol 28: 287-301, 2017.

10. $\mathrm{Li} \mathrm{CH}$ and Chen Y: Targeting long non-coding RNAs in cancers: Progress and prospects. Int J Biochem Cell Biol 45: 1895-1910, 2013.

11. Zhou M, Zhao H, Wang Z, Cheng L, Yang L, Shi H, Yang H and Sun J: Identification and validation of potential prognostic IncRNA biomarkers for predicting survival in patients with multiple myeloma. J Exp Clin Cancer Res 34: 102, 2015.

12. Giulietti M, Righetti A, Principato G and Piva F: IncRNA Co-expression network analysis reveals novel biomarkers for pancreatic cancer. Carcinogenesis 39: 1016-1025, 2018.

13. Ning L, Li Z, Wei D, Chen $\mathrm{H}$ and Yang C: lncRNA, NEAT1 is a prognosis biomarker and regulates cancer progression via epithelial-mesenchymal transition in clear cell renal cell carcinoma. Cancer Biomark 19: 75-83, 2017.

14. White NM, Cabanski CR, Silva-Fisher JM, Dang HX, Govindan R and Maher CA: Transcriptome sequencing reveals altered long intergenic non-coding RNAs in lung cancer. Genome Biol 15: 429, 2014.

15. Liu AN, Qu HJ, Yu CY and Sun P: Knockdown of LINC01614 inhibits lung adenocarcinoma cell progression by up-regulating miR-217 and down-regulating FOXP1. J Cell Mol Med 22: 4034-4044, 2018.

16. Vishnubalaji R, Shaath H, Elkord E and Alajez NM: Long non-coding RNA (lncRNA) transcriptional landscape in breast cancer identifies LINC01614 as non-favorable prognostic biomarker regulated by TGF $\beta$ and focal adhesion kinase (FAK) signaling. Cell Death Discov 5: 109, 2019.

17. Sun $Y$ and Ling C: Analysis of the long non-coding RNA LINC01614 in non-small cell lung cancer. Medicine (Baltimore) 98: e16437, 2019.

18. Wang Y, Song B, Zhu L and Zhang X: Long non-coding RNA, LINC01614 as a potential biomarker for prognostic prediction in breast cancer. PeerJ 7: e7976, 2019.
19. Guo Y, Sheng Q, Li J, Ye F, Samuels DC and Shyr Y: Large scale comparison of gene expression levels by microarrays and RNAseq using TCGA data. PLoS One 8: e71462, 2013.

20. Budczies J, Klauschen F, Sinn BV, Győrffy B, Schmitt WD, Darb-Esfahani S and Denkert C: Cutoff Finder: A comprehensive and straightforward Web application enabling rapid biomarker cutoff optimization. PLoS One 7: e51862, 2012.

21. Livak KJ and Schmittgen TD: Analysis of relative gene expression data using real-time quantitative PCR and the 2(-Delta Delta C(T)) method. Methods 25: 402-408, 2001.

22. Subramanian A, Tamayo P, Mootha VK, Mukherjee S, Ebert BL, Gillette MA, Paulovich A, Pomeroy SL, Golub TR, Lander ES and Mesirov JP: Gene set enrichment analysis: A knowledge-based approach for interpreting genome-wide expression profiles. Proc Natl Acad Sci USA 102: 15545-15550, 2005.

23. Liberzon A, Birger C, Thorvaldsdottir H, Ghandi M, Mesirov JP and Tamayo P: The Molecular Signatures Database (MSigDB) hallmark gene set collection. Cell Syst 1: 417-425, 2015.

24. Duval S and Tweedie R: Trim and fill: A simple funnel-plot-based method of testing and adjusting for publication bias in meta-analysis. Biometrics 56: 455-463, 2000.

25. Iwakiri J, Hamada M and Asai K: Bioinformatics tools for lncRNA research. Biochim Biophys Acta 1859: 23-30, 2016.

26. Bassett AR, Akhtar A, Barlow DP, Bird AP, Brockdorff N, Duboule D, Ephrussi A, Ferguson-Smith AC, Gingeras TR, Haerty W, et al: Considerations when investigating lncRNA function in vivo. ELife 3: e03058, 2014.

27. Joung J, Engreitz JM, Konermann S, Abudayyeh OO, Verdine VK, Aguet F, Gootenberg JS, Sanjana NE, Wright JB, Fulco CP, et al: Genome-scale activation screen identifies a lncRNA locus regulating a gene neighbourhood. Nature 548: 343-346, 2017.

28. Shibayama Y, Fanucchi S, Magagula L and Mhlanga MM: lncRNA and gene looping: What's the connection? Transcription 5: e28658, 2014.

29. Galuschka C, Proynova R, Roth B, Augustin HG and Muller-Decker K: Models in Translational Oncology: A public resource database for preclinical cancer research. Cancer Res 77: 2557-2563, 2017.

30. Kim H, Watkinson J, Varadan V and Anastassiou D: Multi-cancer computational analysis reveals invasion-associated variant of desmoplastic reaction involving INHBA, THBS2 and COL11A1. BMC Med Genomics 3: 51, 2010.

31. Shi JW, Liu W, Zhang TT, Wang SC, Lin XL, Li J, Jia JS, Sheng HF, Yao ZF, Zhao WT, et al: The enforced expression of c-Myc in pig fibroblasts triggers mesenchymal-epithelial transition (MET) via F-actin reorganization and RhoA/Rock pathway inactivation. Cell Cycle 12: 1119-1127, 2013.

32. Zhang B, Zhang C, Yang X, Chen Y, Zhang H, Liu J and Wu Q: Cytoplasmic collagen XI $\alpha$ I as a prognostic biomarker in esophageal squamous cell carcinoma. Cancer Biol Ther 19: 364-372, 2018.

33. Zhu H, Chen H, Wang J, Zhou L and Liu S: Collagen stiffness promoted non-muscle-invasive bladder cancer progression to muscle-invasive bladder cancer. Onco Targets Ther 12: 3441-3457, 2019

34. Liu J, Huang C, Peng C, Xu F, Li Y, Yutaka Y, Xiong B and Yang X: Stromal fibroblast activation protein alpha promotes gastric cancer progression via epithelial-mesenchymal transition through Wnt/ $\beta$-catenin pathway. BMC Cancer 18: 1099, 2018.

35. Yang X, Lin Y, Shi Y, Li B, Liu W, Yin W, Dang Y, Chu Y, Fan J and $\mathrm{He}$ R: FAP promotes immunosuppression by CancerAssociated fibroblasts in the tumor Microenvironment via STAT3-CCL2 signaling. Cancer Res 76: 4124-4135, 2016.

36. Chen L, Tian X, Gong W, Sun B, Li G, Liu D, Guo P, He Y, Chen Z, Xia Y, et al: Periostin mediates epithelial-mesenchymal transition through the MAPK/ERK pathway in hepatoblastoma. Cancer Biol Med 16: 89-100, 2019.

37. Choi KU, Yun JS, Lee IH, Heo SC, Shin SH, Jeon ES, Choi YJ, Suh DS, Yoon MS and Kim JH: Lysophosphatidic acid-induced expression of periostin in stromal cells: Prognoistic relevance of periostin expression in epithelial ovarian cancer. Int J Cancer 128: 332-342, 2011.

38. Shi Q, Bao S, Song L, Wu Q, Bigner DD, Hjelmeland AB and Rich JN: Targeting SPARC expression decreases glioma cellular survival and invasion associated with reduced activities of FAK and ILK kinases. Oncogene 26: 4084-4094, 2007. 
39. Zhang F, Zhang Y, Da J, Jia Z, Wu H and Gu K: Downregulation of SPARC expression decreases cell migration and invasion involving epithelial-mesenchymal transition through the p-FAK/p-ERK pathway in esophageal squamous cell carcinoma. J Cancer 11: 414-420, 2020.

40. Acloque H, Adams MS, Fishwick K, Bronner-Fraser M and Nieto MA: Epithelial-mesenchymal transitions: the importance of changing cell state in development and disease. J Clin Invest 119: 1438-1449, 2009.

41. Thiery JP, Acloque H, Huang RY and Nieto MA Epithelial-mesenchymal transitions in development and disease. Cell 139: 871-890, 2009.

42. Nieto MA: The ins and outs of the epithelial to mesenchymal transition in health and disease. Annu Rev Cell Dev Biol 27: 347-376, 2011.

43. Furuya S, Endo K, Takahashi A, Miyazawa K and Saitoh M: Snail suppresses cellular senescence and promotes fibroblast-led cancer cell invasion. FEBS Open Bio 7: 1586-1597, 2017.
44. Nieto MA: Context-specific roles of EMT programmes in cancer cell dissemination. Nat Cell Biol 19: 416-418, 2017.

45. Lee SJ, Seol HJ, Lee HW, Kang WY, Kang BG, Jin J, Jo MY, Jin Y, Lee JI, Joo KM and Nam DH: Gene silencing of c-Met leads to brain metastasis inhibitory effects. Clin Exp Metastasis 30: 845-854, 2013.

46. Chaffer CL and Weinberg RA: A perspective on cancer cell metastasis. Science 331: 1559-1564, 2011.

47. Jie XX, Zhang XY and Xu CJ: Epithelial-to-mesenchymal transition, circulating tumor cells and cancer metastasis: Mechanisms and clinical applications. Oncotarget 8: 81558-81571, 2017.

This work is licensed under a Creative Commons Attribution-NonCommercial-NoDerivatives 4.0 International (CC BY-NC-ND 4.0) License. 